\title{
Age-Related Metabolic Pathways Changes in Dental Follicles: A Pilot Study
}

Victor Coutinho Bastos ${ }^{1,2}$, Jéssica Gardone Vitório ${ }^{1}$, Roberta Rayra Martins-Chaves ${ }^{1}$, Flávia Leite-Lima ${ }^{1}$, Yuri Abner Rocha Lebron ${ }^{3}$, Victor Rezende Moreira ${ }^{3}$, Filipe Fideles Duarte-Andrade ${ }^{1}$, Thaís dos Santos Fontes Pereira ${ }^{1}$, Lucilaine Valéria de Souza Santos ${ }^{3}$, Liséte Celina Lange ${ }^{3}$, Adriana Nori de Macedo ${ }^{4}$, Gisele André Baptista Canuto ${ }^{5}$, Carolina Cavaliéri Gomes ${ }^{2+}$ and Ricardo Santiago Gomez ${ }^{1 * t}$

OPEN ACCESS

Edited by:

Eliete Neves Da Silva Guerra,

University of Brasilia, Brazil

Reviewed by:

Elena Riet Correa Rivero,

Federal University of Santa

Catarina, Brazil

Rogelio González-González, Juárez University of the State of

Durango, Mexico

Celeste Sanchez-Romero, University of the Republic, Uruguay

*Correspondence: Ricardo Santiago Gomez

rsgomez@ufmg.br

tThese authors have contributed equally to this work and share senior authorship

Specialty section:

This article was submitted to Oral Cancers,

a section of the journal

Frontiers in Oral Health

Received: 08 March 2021

Accepted: 12 May 2021

Published: 18 June 2021

Citation:

Bastos VC, Vitório JG,

Martins-Chaves RR, Leite-Lima F

Lebron YAR, Moreira VR,

Duarte-Andrade FF, Pereira TSF, Santos LVS, Lange LC, Macedo AN,

Canuto GAB, Gomes CC and Gomez RS (2021) Age-Related Metabolic Pathways Changes in Dental Follicles: A Pilot Study. Front. Oral. Health 2:677731 doi: 10.3389/froh.2021.677731
${ }^{1}$ Department of Oral Surgery and Pathology, School of Dentistry, Universidade Federal de Minas Gerais, Belo Horizonte, Brazil, ${ }^{2}$ Department of Pathology, Biological Sciences Institute, Universidade Federal de Minas Gerais, Belo Horizonte, Brazil, ${ }^{3}$ Department of Sanitary and Environmental Engineering, School of Engineering, Universidade Federal de Minas Gerais, Belo Horizonte, Brazil, ${ }^{4}$ Department of Chemistry, Exact Sciences Institute, Universidade Federal de Minas Gerais, Belo Horizonte, Brazil, ${ }^{5}$ Department of Analytical Chemistry, Institute of Chemistry, Universidade Federal da Bahia, Salvador, Brazil

Aging is not a matter of choice; it is our fate. The "time-dependent functional decline that affects most living organisms" is coupled with several alterations in cellular processes, such as cell senescence, epigenetic alterations, genomic instability, stem cell exhaustion, among others. Age-related morphological changes in dental follicles have been investigated for decades, mainly motivated by the fact that cysts and tumors may arise in association with unerupted and/or impacted teeth. The more we understand the physiology of dental follicles, the more we are able to contextualize biological events that can be associated with the occurrence of odontogenic lesions, whose incidence increases with age. Thus, our objective was to assess age-related changes in metabolic pathways of dental follicles associated with unerupted/impacted mandibular third molars from young and adult individuals. For this purpose, a convenience sample of formalin-fixed paraffin-embedded (FFPE) dental follicles from young ( $<16$ y.o., $n=13$ ) and adult (>26 y.o., $n=7$ ) individuals was selected. Samples were analyzed by high-performance liquid chromatography-mass spectrometry (HPLC-MS)-based untargeted metabolomics. Multivariate and univariate analyses were conducted, and the prediction of altered pathways was performed by mummichog and Gene Set Enrichment Analysis (GSEA) approaches. Dental follicles from young and older individuals showed differences in pathways related to C21-steroid hormone biosynthesis, bile acid biosynthesis, galactose metabolism, androgen and estrogen biosynthesis, starch and sucrose metabolism, and lipoate metabolism. We conclude that metabolic pathways differences related to aging were observed between dental follicles from young and adult individuals. Our findings support that similar to other human tissues, dental follicles associated with unerupted tooth show alterations at a metabolic level with aging, which can pave the way for further studies on oral pathology, oral biology, and physiology.

Keywords: aging, dental follicle, dental sac, developmental biology, oral pathology, untargeted metabolomics, LC-MS 


\section{INTRODUCTION}

Dental follicles, also referred to as dental sacs, comprise a defined structure with a remarkable role in periodontogenesis and tooth eruption [1-4]. Classically, the definition of dental follicle includes the ectomesenchymal tissue derived from the neural crest that involves the tooth during development (i.e., odontogenesis) [5]. By a surgical definition, however, the term "dental follicle" comprises both the ectomesenchymal tissue around the developing tooth as well as the reduced enamel epithelium [6]. In cases when tooth eruption does not occur, the dental follicle remains attached to the coronal portion of the unerupted or impacted tooth [7]. Some pathological changes are described in this context, and are often agedependent, as in the case of squamous and mucous cell metaplasia/prosoplasia. They involve molecular changes, such as increased expression of cell proliferation markers and lower expression of pro-apoptotic markers, mainly attributed to the epithelial component of this dental sac [8-12]. Some of these molecular and morphological alterations are considered as early signs of events that give rise to odontogenic lesions, such as developmental cysts and tumors. However, this is known to be a rare event [13].

Aging is a complex process that involves alterations in many cellular processes resulting in/from altered cell-cell interactions, loss of cell proteostasis, telomeric shortening, cell senescence, mitochondrial dysfunction, epigenetic alterations, genomic instability, stem cell exhaustion, among others [14]. The multi-level complexity underlying the aging process involves interactions between nucleic acids and proteins and is also influenced by environmental factors, constituting the aging phenotype [15]. Metabolomics is an emerging field among the so-called "-omics sciences" and correspond to a promising tool to understand the phenotype of cells, tissues, organs, and organisms in different conditions [16-18]. Thus, by exploring the metabolome, set of all metabolites in complex organisms, phenotypic changes related to biological functions can be revealed, especially when subtle changes in the concentration of metabolites can be targeted [19]. Some effective studies have been conducted in this context, unveiling the impact of aging on different tissues [15]. By exploring the aging physiology, the course of diseases in which the incidence increases with age, such as Alzheimer's disease, rheumatic disorders, cardiovascular, metabolic diseases, and, a prominent example, cancer, can be understood [20].

In the present study, we investigated the altered metabolic pathways in dental follicles of third-molar teeth from young and adult individuals by an untargeted metabolomics approach. We aimed to provide a list of predicted altered pathways, which can pave the way for further research that may use dental follicles/dental sacs as a model to understand aging, tooth eruption, bone physiology, and epithelium-mesenchymal interactions, as well as other phenomena that can be assessed in this peculiar tissue.

\section{MATERIALS AND METHODS}

\section{Patients and Tissue Specimens}

A convenience sample $(n=20)$ of formalin-fixed paraffinembedded (FFPE) tissue samples was retrieved from the archives of the Oral Pathology Service at Universidade Federal de Minas Gerais (UFMG, Brazil). Dental sacs associated with impacted/completely unerupted mandibular third molars with diagnosis confirmed by the histopathological analysis were used. The histopathological diagnosis of all samples was confirmed by three investigators (RSG, CCG, and VCB). After searching the files and retrieving the samples from the archives, we sought to select the best cases from the youngest and the oldest patients possible, based on tissue availability as well as histological features. Thus, samples showing intense inflammatory infiltrate or bony trabeculae were excluded since both could potentially affect the findings and/or metabolite extraction protocol. Samples with the clinical diagnosis of odontogenic cysts were also excluded.

\section{Sample Preparation}

Briefly, $\sim 10 \mathrm{mg}$ of tissue was obtained from FFPE specimens. Thick tissue sections of $20 \mu \mathrm{m}$ were obtained using a manual microtome and collected into previously weighed microfuge tubes. The first two sections were discharged to remove potential surface contaminants. Tissues were then deparaffinized by four sequential incubations with cold xylene (Merck, Darmstadt, HE, Germany), each followed by centrifugations $\left(15,000 \times g, 15 \mathrm{~min}, 4^{\circ} \mathrm{C}\right)$. Residual solvent was dried using a dry bath incubator at $37^{\circ} \mathrm{C}$ and then weighed. The metabolite extraction solution was composed of a mixture of HPLC grade methanol:water:chloroform (3:1:1, v/v/v) (Sigma-Aldrich, San Luis, MO, USA). The volume of extraction solution was normalized per biomass of each tissue pellet $(1 \mathrm{mg}: 20 \mu \mathrm{L}$ of solution). Samples were homogenized using an ultrasonic bath for $10 \mathrm{~min}$ and centrifuged. The supernatant was filtered in nylon syringe-filters ( $13 \mathrm{~mm}$ diameter, $0.22 \mu \mathrm{m}$ pore size), collected into inserts in glass vials, and sealed after sample preparation. A quality control (QC) sample was prepared by pooling $10 \mu \mathrm{L}$ of each sample. Samples were stored at $-80^{\circ} \mathrm{C}$ until analysis.

\section{High-Performance Liquid Chromatography-Mass Spectrometry}

Extracts were examined by HPLC-MS (Shimadzu HPLC System, LC 20A, Kyoto, Japan) coupled with a Bruker Quadrupole Timeof-Flight (QToF) mass spectrometer (Bruker micrOTOF QII, Billerica, MA, USA). A reverse phase, $\mathrm{C}_{18}, 5 \mathrm{~cm} \times 2.1 \mathrm{~mm}$ i.d., $1.9 \mu \mathrm{m}$ chromatography column (Supelco Discovery HS, Bellefonte, PA, USA) was used for metabolite separation at $40^{\circ} \mathrm{C}$. The HPLC was operated according to the following parameters: injection volume $=10 \mu \mathrm{L}$; flow rate $=0.3 \mathrm{~mL} / \mathrm{min}$. HPLC solvents were taken as follows: A, MilliQ Water with $0.1 \%(\mathrm{v} / \mathrm{v})$ formic acid; and B, acetonitrile (ACN, Sigma-Aldrich, San Luis, MO, USA) with $0.1 \%$ (v/v) formic acid (Sigma-Aldrich, San Luis, MO, USA) for positive ionization mode; and A, MilliQ water; 
and $\mathrm{B}, \mathrm{ACN}$ for negative ionization mode. The separation was conducted with gradient mode, in which the elution range was given as follows: $25-95 \% \mathrm{~B}$ (20 $\mathrm{min})$; $95 \% \mathrm{~B}$ (3 $\mathrm{min})$; $95-20 \% \mathrm{~B}$ ( $2 \mathrm{~min}$ ); and $20 \%$ B for $5 \mathrm{~min}$. Mass spectra were acquired using positive and negative-mode electrospray ionization (ESI+ and ESI-, respectively). The capillary voltage was $4,500 \mathrm{~V}$ for ESI+ and 3,500 V for ESI-. Nitrogen was used as cone and desolvation gas with a pressure of 2 bar and a flow rate of $7.0 \mathrm{~L} / \mathrm{h}$. The source temperature was $100^{\circ} \mathrm{C}$, and the desolvation temperature was $180^{\circ} \mathrm{C}$. Nitrogen was also used as collision gas and it was generated by a nitrogen generator (NM32LA, Peak Scientific, SP, Brazil). A full scan from $90-1200 \mathrm{~m} / \mathrm{z}$ was obtained using sodium formate as calibrator. The QC sample was reinjected multiple times after each five sequential sample injections.

\section{Data Preprocessing}

Raw data were converted into .mzXML open format with MSConvert software (ProteoWizard, v.3.0). After conversion, optimization of parameters for data treatment was performed using QC data files. Data were optimized separately for ESI+ and ESI- in IPO package version 1.16.0 (Libiseller et al., 2015) in R Statistic workplace ( $\mathrm{R}$ version 3.6.2). A two-step workflow was employed: the first consisted of optimization of initial default XCMS parameters followed by optimization of the whole set of parameters for peak picking and retgroup functions. We edited the minfrac and minsample parameters (set to 0.7 and 2, respectively), since they contribute to the concordance of $\mathrm{m} / \mathrm{z}$ features within each group, increasing analytical robustness [21]. Optimized values of each parameter are shown in Supplementary File 1. Files were then processed on the XCMS package [22] in R Statistical workplace. Features extracted at $<2.5 \mathrm{~min}$ and $>20 \mathrm{~min}$ were removed. Duplicity was also checked by Pearson's correlation of features coeluted within a $2.5 \mathrm{~min}$ interval, considering a $5 \mathrm{ppm}$ mass error (for this purpose, $r>0.7$ correlation of peak intensities were considered as features duplicities and were removed from the data matrix). After this preprocessing step, the data matrix consisted of 3,530 and $475 \mathrm{~m} / z$ features, in ESI+ and ESI- modes, respectively.

\section{Data Processing and Statistical Analysis}

Multivariate analysis was conducted on MetaboAnalyst 4.0 online platform $[23,24]$. Principal Component Analysis (PCA) and Partial Least Square Discriminant Analysis (PLS-DA) models were constructed based on data input after data filtering and missing value imputation. Features presenting more than $20 \%$ of missing values were excluded, and the residual missing values were replaced by the half of minimum value of intensity for each feature; data filtering based on relative SD (features presenting $\mathrm{RSD}>25 \%$ for the peak intensities in the QCs were excluded) and interquartile range (IQR), eliminating features with poor analytical consistency and non-informative variables. Data were then median normalized, log-transformed, and Pareto-scaled for multivariate analysis. For univariate statistical analysis, the same filters and normalizations were applied except for the Pareto scaling. Shapiro-Wilk normality test, followed by Independent Samples $t$-Test (Welch's $t$-Test) (accounting for Levene's test for equality of variances) or Mann-Whitney U Tests were conducted on IBM SPSS Statistics 26 (IBM Corporation, v.26.0.0.0) when applicable. False Discovery Rate (FDR) was applied to employ the Benjamini-Hochberg method and a cutoff of $5 \%$ was considered.

\section{Pathway Enrichment}

As a functional interpretation of global metabolomics data can be challenging [25], we used the MetaboAnalyst 4.0 module called "MS Peaks to Pathways," which integrates mummichog and Gene Set Enrichment Analysis (GSEA) algorithms to predict changes in metabolic pathways in given conditions [26]. Mummichog [27] is an algorithm based on Over Representation Analysis (ORA), which assumes that a certain degree of random errors during individual peak assignment will not change the collective behavior jointly determined by all metabolites involved in the pathways [28]. This algorithm tests if statistically significant peaks of a given list are enriched compared with null models drawn from the user input list. GSEA [29] is a cutoff-free method that evaluates the overall differences between two distributions based on Kolmogorov-Smirnov tests and favors the detection of subtle and consistent alterations that can be lost by ORA approaches.

For this purpose, a five-column table was built consisting data of 2,068 remaining features retained after data filtering of MetaboAnalyst for both ionization modes. The input table presented the headers " $m . z$ " for mass-to-charge ratios; " $r . t$ " for retention time; " $p$-values" from both Independent Samples $t$-Test or Mann-Whitney $U$ Test (raw $p$-values, non-FDR corrected); "mode" indicating the ionization mode (positive or negative); and "t.score" calculated for each feature. A mass error of 10 ppm was used since it seemed more reasonable with QToF mass resolution, as $M$ s peaks to Pathways developers encourage inputs derived from high-resolution analytical platforms, such as Orbitrap or other Fourier transform mass spectrometry family. For mummichog analysis, a $p$-value cutoff of 0.05 was used. The library selected was the Human MFN Model, which is manually curated and originated from different sources (KEGG, BiGG, and Edinburgh Model) [30].

\section{RESULTS}

\section{Sample Characterization}

Thirteen samples were included in the young group of patients (13-16 years old, median $=15$ years old), and seven in the adult group $(26-38$ years old, median $=28$ years old). Although we originally intended to have equal numbers of samples in both groups, samples from dental follicles of older patients were difficult to obtain, as most of the surgical procedures to remove unerupted thirds molars occur in adolescents. The young group was comprised of six boys and seven girls. Four females and three males formed the adult group. The main histological findings are shown in Figure 1. Not all samples exhibited evident epithelial lining, but the presence of islets of the odontogenic epithelium was frequently observed, especially among younger individuals (Figure 1c). We observed the presence of reduced enamel epithelium in $6 / 13$ samples in the young group of patients. Squamous epithelium was present in 3/7 samples among 

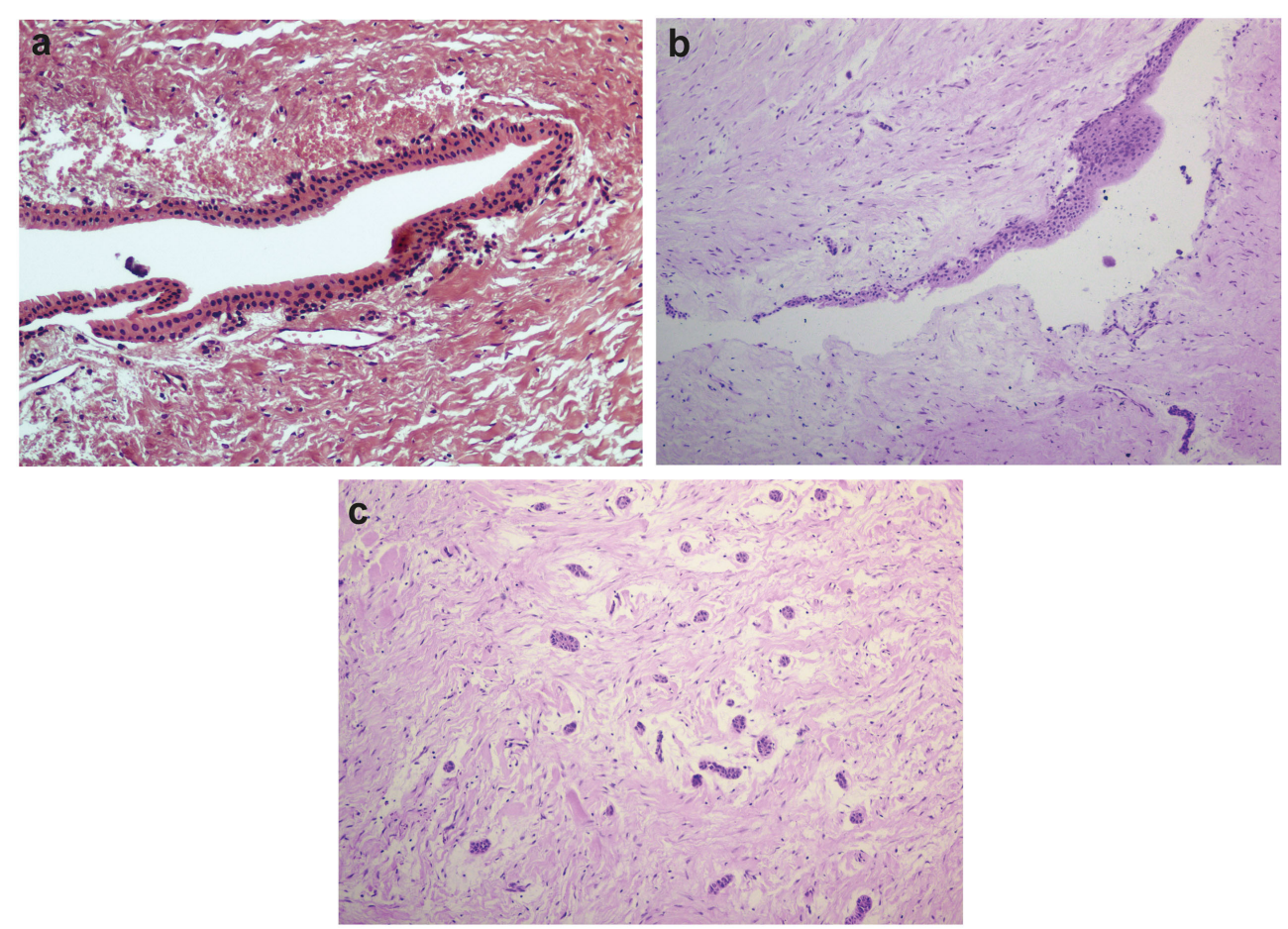

FIGURE 1 | Histological features of dental follicles associated with unerupted mandibular third molars. (a) Reduced enamel epithelium lining connective tissue of dental follicle (x20 magnification). (b) Stratified squamous epithelial lining with focal thickening (x10 magnification). (c) Typical islets of the odontogenic epithelium were commonly observed in dental follicles from young individuals (x10 magnification). Slides were hematoxylin-eosin stained.

adult individuals and was also present in focal areas of $2 / 13$ samples from young patients.

\section{Quality Assessment and Statistical Evaluation}

Principal Component Analysis indicated good analytical stability during the HPLC-MS run, for both positive and negative ionization modes (Supplementary File 2). PLS-DA analysis showed some degree of group separation, but the generated models could not be interpreted as predictive and were considered overfitted (Supplementary File 2). The univariate analysis resulted in a total of 287 molecular features statistically significant ( $p<0.05$, non-significant after FDR correction) from a total of 2,068 features in both positive and negative ionization modes. Only two $\mathrm{m} / z$ features were retained after $5 \%$ correction of FDR $(m / z$ 647.2999, adjusted $p$-value $<0.0001 ; m / z 379.1334$, adjusted $p$-value $<0.0001$, both detected on ESI+ mode). Since a single $m / z$ feature can match multiple compounds [27], they were not individually examined.

\section{Pathway Enrichment}

We carried out pathway enrichment based on mummichog and its integration with the GSEA approach, currently available within the "MS peaks to pathways" module of MetaboAnalyst 4.0. It is important to emphasize that the prediction algorithms were solely used as tools to identify metabolic pathways, with no purpose of annotating metabolites. Mummichog v.2 was used, and it includes retention time information to increase the confidence and robustness of potential compound matches [30], thus resulting in 248 matched compounds (Supplementary File 3). By executing the same analysis without retention time information (mummichog v.1), 1,355 compounds were matched and resulted pathways were reported (Supplementary File 4). For the sake of clarity, the discussion will be based on the results of the analysis that accounted for retention time analysis, although we cannot entirely discharge any other results. This analysis identified several pathways, including the C-21 steroid hormone biosynthesis, the bile acid biosynthesis, the galactose metabolism, the androgen and estrogen metabolism, the starch and sucrose and lipoate metabolic pathways (Gamma-p-values < 0.05) in mummichog approach. Tables 1, 2 provide the results from the mummichog approach alone and integrated with GSEA, respectively. Figure 2 shows scatter plots derived from each analysis.

\section{DISCUSSION}

In the present study, we aimed to assess the metabolic alterations related to aging in dental sacs of impacted or unerupted mandibular third molars. These are the teeth that are the most frequently associated with odontogenic lesions development [13, 31 ] and are the last tooth to achieve occlusal plane, generally erupting between 17 and 26 years [13]. Considering that most of the third molar extraction surgeries are performed at a 
TABLE 1 | Results of the mummichog pathway analysis.

\begin{tabular}{|c|c|c|c|c|c|c|c|}
\hline Enriched pathways & Pathway total & Hits.total & Hits.sig. & Expected & FET & EASE & Gamma-p value \\
\hline $\begin{array}{l}\text { C21-steroid hormone biosynthesis } \\
\text { and metabolism }\end{array}$ & 8 & 8 & 4 & 1.0256 & 0.0066 & 0.0423 & 0.0436 \\
\hline Bile acid biosynthesis & 11 & 11 & 4 & 1.4103 & 0.0250 & 0.1016 & 0.0443 \\
\hline Galactose metabolism & 3 & 3 & 2 & 0.3846 & 0.0360 & 0.2956 & 0.0447 \\
\hline $\begin{array}{l}\text { Androgen and estrogen } \\
\text { biosynthesis and metabolism }\end{array}$ & 3 & 3 & 2 & 0.3846 & 0.0360 & 0.2956 & 0.0447 \\
\hline Starch and Sucrose Metabolism & 3 & 3 & 2 & 0.3846 & 0.0360 & 0.2956 & 0.0447 \\
\hline Lipoate metabolism & 1 & 1 & 1 & 0.1282 & 0.1171 & 1 & 0.0477 \\
\hline $\begin{array}{l}\text { Vitamin D3 (cholecalciferol) } \\
\text { metabolism }\end{array}$ & 2 & 2 & 1 & 0.2564 & 0.2214 & 1 & 0.0522 \\
\hline Vitamin E metabolism & 3 & 3 & 1 & 0.3846 & 0.3141 & 1 & 0.0569 \\
\hline Fatty acid metabolism & 4 & 4 & 1 & 0.5128 & 0.3964 & 1 & 0.0616 \\
\hline Linoleate metabolism & 7 & 7 & 1 & 0.8974 & 0.5914 & 1 & 0.0766 \\
\hline $\begin{array}{l}\text { Prostaglandin formation from } \\
\text { arachidonate }\end{array}$ & 8 & 8 & 1 & 1.0256 & 0.6420 & 1 & 0.0818 \\
\hline Glycosphingolipid metabolism & 9 & 9 & 1 & 1.1538 & 0.6868 & 1 & 0.0871 \\
\hline
\end{tabular}

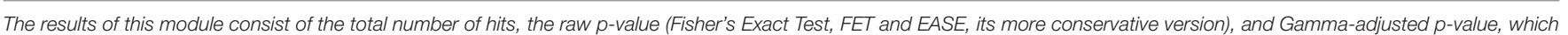

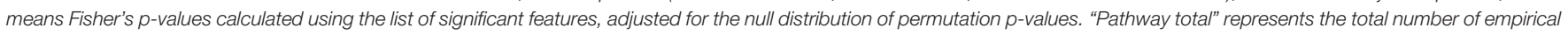

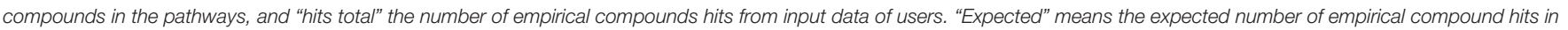
the pathway.

TABLE 2 | Integrated results of the mummichog and Gene Set Enrichment Analysis (GSEA) Pathway.

\begin{tabular}{|c|c|c|c|c|c|c|}
\hline Enriched Pathways & Total Size & Hits & Hits Sig. & Mummichog $p$ values & GSEA $p$ values & Combined $p$ values \\
\hline Galactose metabolism & 3 & 3 & 2 & 0.0360 & 0.0714 & 0.0179 \\
\hline Starch and Sucrose Metabolism & 3 & 3 & 2 & 0.0360 & 0.0714 & 0.0179 \\
\hline C21-steroid hormone biosynthesis and metabolism & 8 & 8 & 4 & 0.0066 & 0.4362 & 0.0198 \\
\hline Bile acid biosynthesis & 11 & 11 & 4 & 0.0250 & 0.1979 & 0.0313 \\
\hline Androgen and estrogen biosynthesis and metabolism & 3 & 3 & 2 & 0.0360 & 0.7143 & 0.1201 \\
\hline Vitamin D3 (cholecalciferol) metabolism & 2 & 2 & 1 & 0.2215 & 0.2698 & 0.2281 \\
\hline Lipoate metabolism & 1 & 1 & 1 & 0.1172 & 0.5192 & 0.2312 \\
\hline Prostaglandin formation from arachidonate & 8 & 8 & 1 & 0.6421 & 0.125 & 0.2827 \\
\hline Fatty Acid Metabolism & 4 & 4 & 1 & 0.3964 & 0.4286 & 0.4711 \\
\hline Vitamin E metabolism & 3 & 3 & 1 & 0.3141 & 0.6857 & 0.5461 \\
\hline Glycosphingolipid metabolism & 9 & 9 & 1 & 0.6868 & 0.3656 & 0.5981 \\
\hline Linoleate metabolism & 7 & 7 & 1 & 0.5914 & 0.5568 & 0.6951 \\
\hline
\end{tabular}

GSEA considers the overall ranks of features without using a significant cutoff. The module uses Fisher's method to combine raw p-values of mummichog and GSEA approaches.

younger age [32, 33], it was difficult to retrieve dental follicle samples from patients above 30 years old. We observed the presence of reduced enamel epithelium in 6/13 samples in the young group of patients. Typical small islets and strands of odontogenic epithelium were most commonly observed in the young group, agreeing with the literature [7]. Squamous epithelium was present in 3/7 samples among adult individuals. If squamous metaplasia of the reduced enamel epithelium can represent an early sign of pathological change in dental follicles is debatable [8-12, 34], as tissues can naturally undergo morphological changes to adapt to new circumstances [7, 35-38]. The relevance of this debate relies on the absence of a consensus in the clinical practice guidelines justifying prophylactic surgical removal of asymptomatic disease-free impacted teeth [13].
Differences between the young and adult dental follicle groups were observed in many metabolic pathways, such as the C21-steroid hormone biosynthesis and metabolism, the bile acid biosynthesis, the galactose metabolism, the androgen and estrogen biosynthesis and metabolism, the starch and sucrose metabolism, and the lipoate metabolism. Although we were not able to observe which metabolic pathways had decreased or increased activities between experimental groups, these metabolic pathways were already described in the aging context and will be briefly discussed. It is important to point out that puberty is, likewise, a complex process coupled to several hormonal and physiological changes [39], which must be kept in mind when dealing with metabolomic studies assessing young patients and may explain, for example, the fact that we found differences in androgen and estrogen biosynthesis and 
metabolism between our experimental groups. We encourage further validation studies on larger sample size and based on targeted approaches.

C21-steroid hormone biosynthesis and metabolism comprises progesterone-aldosterone and progesterone-cortisol/cortisone axes that mediate a wide variety of biological processes such as reproductive function, sexual development, electrolyte balance, blood pressure, and stress responses [40]. Steroidal metabolome was previously demonstrated to be influenced by sex, age, and circadian cycle [40-42].

Bile acids (BAs) comprise a group of important physiological agents for cholesterol metabolism, intestinal nutrient absorption, biliary secretion of lipids, toxic metabolites, and xenobiotics [43]. Through activation of signaling pathways triggered by the activation of G-protein-coupled receptors (GPCRs) or nuclear bile acid receptors (including farnesoid-X receptor, FXR, also known as NR1H4), bile acids have been shown to regulate not only their own synthesis and enterohepatic recirculation, but also regulate triglyceride, cholesterol, energy, and glucose homeostasis [44]. Changes in serum and blood plasma levels were already found to be age-related and sex-specific [43, 45]. Interestingly, genes related to the bile secretion pathway were reported to act in tooth germs development of rats at RNA and protein levels [46].

The main pathway of galactose metabolism in humans involves the conversion of galactose into glucose by galactokinase and galactose-1-phosphate uridyl-transferase for glycolysis [47, 48]. In animal models, long-term D-galactose exposure induces the acquisition of an aging phenotype, which has been recognized as being similar to those in naturally aged mice and rats [49]. Ageinduced animals exhibit increased reactive oxygen species (ROS) formation and decreased antioxidant enzyme activity in the brain, poor immune responses, cognitive dysfunctions, weakened motor function, and shortened lifespan [48, 50], mainly the effects of the accumulation of senescent cells in naturally aged organisms [20,51-53]. In these models, the impacts of impaired galactose metabolism have already been studied on the brain, liver, lungs, heart, kidney, skin, reproductive systems, and others [54]. Other carbohydrates cause oxidative stress by activation of mitochondrial metabolism of glucose, leading to ROS generation [55]. In this context, starch and sucrose metabolism may also be related. In this case, ROS is generated through mitochondrial respiratory chain enzymes, xanthine oxidases, lipoxygenases, cyclooxygenases, nitric oxide synthases, and peroxidases [55].

Lipoate metabolism plays a key role in mitochondrial functions [56]. Lipoate is a covalently bound cofactor essential for five redox reactions in humans: four 2-oxoacid dehydrogenases and the glycine cleavage system (GCS). Two enzymes are derived from the energy metabolism, $\alpha$-ketoglutarate dehydrogenase and pyruvate dehydrogenase; and three are derived from the amino acid metabolism, branched-chain ketoacid dehydrogenase, 2oxoadipate dehydrogenase, and the GCS [57]. Lipoate is the conjugate base of lipoic acid (LA), and the most prevalent form of LA under physiological conditions. It presents a highly negative reduction potential, increases the expression

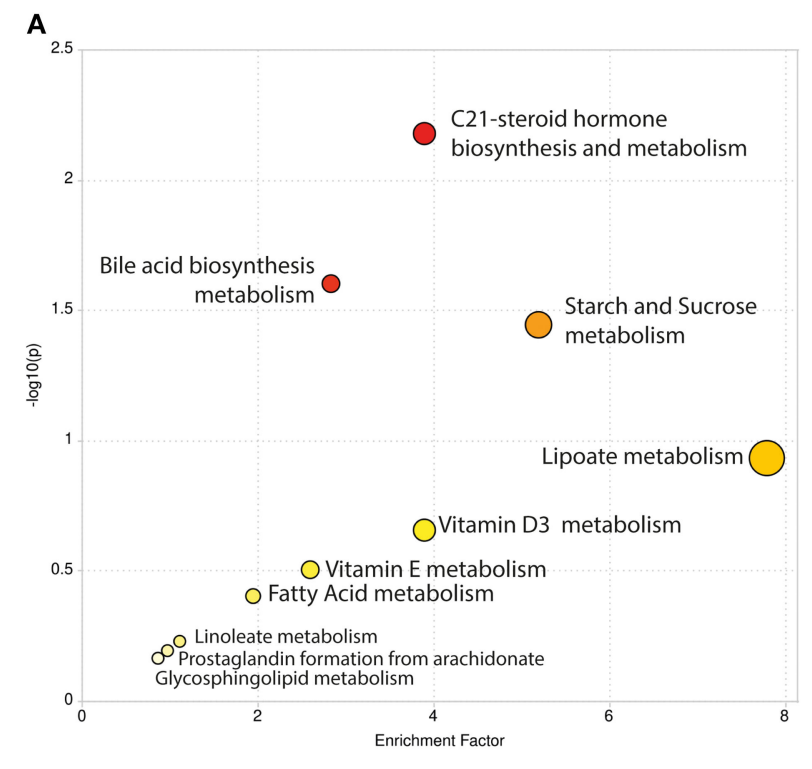

B

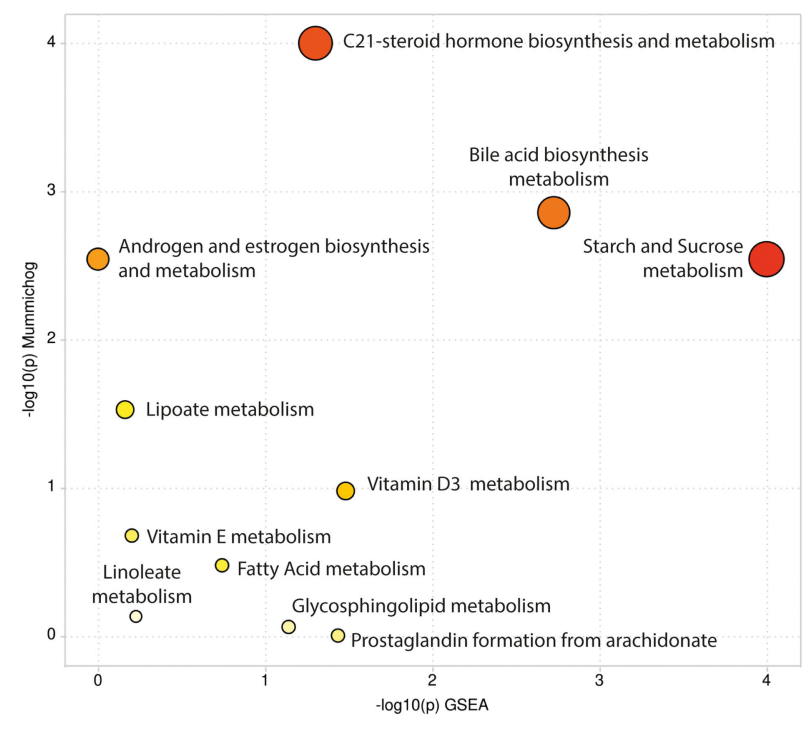

FIGURE 2 | Scatter Plots of Pathway Enrichment Analysis provided by mummichog (A) and by its integration with GSEA (B). The color and size of each circle correspond to its $p$-value and enrichment factor, respectively. Darker tones indicate more statistically relevant predicted pathways. The size of each dot represents the ratio between significant pathway hits and the expected number of compound hits within the pathways.

of antioxidant enzymes, and participates in the recycling of vitamins C and E. Due to these properties, LA is called the "universal antioxidant" [58]. LA displays anti-apoptotic and anti-inflammatory properties in in vivo and in vitro studies. Importantly, it has been shown that LA reverses the ageassociated loss of neurotransmitters and their receptors, which can underlie its effects on cognitive functions [59].

Cellular, genetic, endocrine, molecular, and environmental factors were also involved in tooth eruption (as we are dealing 
with unerupted and impacted teeth) and were not entirely covered in our study design. Nutritional status, body mass index (BMI), socioeconomic status, and others should be stratified in further studies with larger sample size, since these are important factors affecting the metabolome and, accordingly, tooth eruption [60-63]. Moreover, causes of primary failure of eruption or delayed tooth eruption are not fully understood, but disruptions and/or total failure in the ability of dental follicles leading to bone resorption throughout the gubernacular canal are discussed [60, 61, 64]. From this perspective, metabolic alterations in dental follicles may lead tooth eruption to fail and should be explored.

The more deeply we understand tissue physiology, the more we become capable of reframing several biological questions, changing the status quo, and casting light into new perspectives. Recently, from genetic approaches, it has been observed that normal, pathology-free tissues also can harbor pathological mutations, some of which are known oncogenes, implicating a new way to look at genetic alterations [65-67]. Benign tumors often can exhibit hotspot mutations and still present indolent clinical behavior [67-69]. Regarding dental follicles, no hotspot mutations were found until now [70]. Global profiling of DNA methylation and hydroxymethylation was also recently explored, and, despite age-related decrease of global DNA hydroxymethylation found, the biological meaning of this epigenetic profile change in dental follicles remains to be elucidated [71].

Genotype-phenotype interactions are complex, and many variables can elicit different responses at cells, tissues, and organisms. Metabolomics can bridge the gap, making paradoxes to be reconciled. Recently, metabolism from a wide variety of sources (e.g., alcohol and microbial metabolism) was found to modify DNA and histones and exerts specific effects on cell biology, systemic physiology, and pathology [72]. Polyphenism, a peculiar sub-type of phenotypic plasticity present in several animal species were ultimately proposed to happen in humans at a metabolic level [73]. In our study, the C21-steroid hormone biosynthesis, the bile acid biosynthesis, the galactose metabolism, the androgen and estrogen biosynthesis, the starch and sucrose and lipoate metabolism have been found to correlate to aging in dental sacs.

It is important to note that our predictions of altered metabolic pathways were performed based on untargeted HPLCMS-based metabolomics of FFPE tissue samples, which has just recently become appreciated in the metabolomics field, inspiring protocols to be developed and optimized [74-76]. In this provocative and hypothesis-generator study, we shed light on few aspects of dental follicles physiology and so many others must be discovered. Dental follicles of unerupted/impacted teeth (i.e., dental sacs) are a unique tissue in the human body, hermetically encased within the alveolar bone. They are less exposed to exogenous environmental agents and are a reminiscence of tissue interactions dating back to early odontogenesis. We encourage scientists to consider this valuable tissue as a unique model and to explore its potential to answer a wide range of questions in the fields of physiology, developmental biology, and pathology.

\section{DATA AVAILABILITY STATEMENT}

The original contributions generated for this study are included in the article/Supplementary Material, further inquiries can be directed to the corresponding author/s.

\section{ETHICS STATEMENT}

The studies involving human participants were reviewed and approved by Ethics Committee of Universidade Federal de Minas Gerais (UFMG, Brazil) (protocol number/approval: 30697120.9.0000.5149/4.082.478). Written informed consent to participate in this study was provided by the participants' legal guardian/next of kin.

\section{AUTHOR CONTRIBUTIONS}

VB, JV, TP, FD-A, YL, and VM performed the experiments. $\mathrm{VB}, \mathrm{JV}, \mathrm{RM}-\mathrm{C}$, and FL-L performed the bioinformatic analyses. $\mathrm{VB}$, JV, and RG performed data analyses. VB drafted the manuscript and generated the text and figures. LS and LL provided resources for HPLC-MS instrumentation. AM, GC, CG, and RG critically revised the manuscript. RG and CG provided leadership for the project. All authors contributed to the final manuscript.

\section{FUNDING}

This study was financed in part by the Coordination for the Improvement of Higher Education Personnel (CAPES)/Brazil [finance code 001] and by the Research Support Foundation of the State of Minas Gerais (FAPEMIG)/Brazil [grant number RED 00019-16]. VB, FD-A, RM-C, and TP received CAPES scholarship, and JV, YL, and VM received a National Council for Scientific and Technological Development $(\mathrm{CNPq}) /$ Brazil scholarship. CG and RG are research fellows at $\mathrm{CNPq} /$ Brazil.

\section{ACKNOWLEDGMENTS}

We would like to thank the entire staff of Oral Pathology Service (UFMG, Brazil).

\section{SUPPLEMENTARY MATERIAL}

The Supplementary Material for this article can be found online at: https://www.frontiersin.org/articles/10.3389/froh. 2021.677731/full\#supplementary-material 


\section{REFERENCES}

1. Cho M-I, Garant PR. Development and general structure of the periodontium. Periodontol. (2000) 24:9-27. doi: 10.1034/j.1600-0757.2000.2240102.x

2. Cahill DR, Marks SC. Tooth eruption: evidence for the central role of the dental follicle. J Oral Pathol Med. (1980) 9:189200. doi: $10.1111 /$ j.1600-0714.1980.tb00377.x

3. Marks SCJ. The basic and applied biology of tooth eruption. Connect Tissue Res. (1995) 32:149-57. doi: 10.3109/03008209509013718

4. Sarrafpour B, Swain M, Li Q, Zoellner H. Tooth eruption results from bone remodelling driven by bite forces sensed by soft tissue dental follicles: a finite element analysis. PLoS ONE. (2013) 8:e58803. doi: 10.1371/journal.pone.0058803

5. Cate AR Ten, Mills C, Solomon G. The development of the periodontium. A transplantation and autoradiographic study. Anat Rec. (1971) 170:36579. doi: $10.1002 /$ ar. 1091700312

6. Larson EK, Cahill DR, Gorski JP, Marks SC. The effect of removing the true dental follicle on premolar eruption in the dog. Arch Oral Biol. (1994) 39:271-5. doi: 10.1016/0003-9969(94)90116-3

7. Stanley HR, Krogh H, Pannkuk E. Age changes in the epithelial components of follicles (dental sacs) associated with impacted third molars. Oral Surg Oral Med Oral Pathol. (1965) 19:128-39. doi: 10.1016/0030-4220(65)90226-4

8. Cabbar F, Güler N, Comunoglu N, Sençift K, Çöloglu S. Determination of potential cellular proliferation in the odontogenic epithelia of the dental follicle of the asymptomatic impacted third molars. J Oral Maxillofac Surg. (2008) 66:2004-11. doi: 10.1016/j.joms.2008.06.011

9. Coşarcă AS, Mocan SL, Păcurar M, Fülöp E, Ormenişan A. The evaluation of Ki67, p53, MCM3 and PCNA immunoexpressions at the level of the dental follicle of impacted teeth, dentigerous cysts and keratocystic odontogenic tumors. Rom J Morphol Embryol. (2016) 57:407-12.

10. Kucukkolbasi H, Esen A, Erinanc O. Immunohistochemical analysis of Ki-67 in dental follicle of asymptomatic impacted third molars. J Oral Maxillofac Pathol. (2014) 18:189-93. doi: 10.4103/0973-029X.140737

11. Matsumoto MA, Filho HN, Jorge FM, Salvadori DMF, Marques MEA, Ribeiro DA. Expression of cell cycle regulatory proteins in epithelial components of dental follicles. J Mol Histol. (2006). 37:127-31. doi: 10.1007/s10735-006-9048-3

12. Razavi SM, Hasheminia D, Mehdizade M, Movahedian B, Keshani F. The relation of pericoronal third molar follicle dimension and bcl-2/ki67 expression: an immunohistochemical study. Dent Res J. (2012) 9:S2631. doi: 10.4103/1735-3327.107931

13. Ghaeminia H, Nienhuijs MEL, Toedtling V, Perry J, Tummers M, Hoppenreijs TJ, et al. Surgical removal versus retention for the management of asymptomatic disease-free impacted wisdom teeth. Cochrane Database Syst Rev. (2020) 5:CD003879. doi: 10.1002/14651858.CD003879.pub5

14. López-Otín C, Blasco MA, Partridge L, Serrano M, Kroemer G. The hallmarks of aging. Cell. (2013) 153:1194-217. doi: 10.1016/j.cell.2013.05.039

15. Srivastava S. Emerging insights into the metabolic alterations in aging using metabolomics. Metabolites. (2019) 9:301. doi: 10.3390/metabo9120301

16. Nalbantoglu S. Metabolomics: basic principles and strategies. In: Nalbantoglu S, Amri H, editors. Molecular Medicine. Rijeka, Croatia: IntechOpen. p. 1-15. doi: 10.5772/intechopen. 88563

17. Fiehn O. Metabolomics - the link between genotypes and phenotypes. In: Town C, editor. Functional Genomics. Dordrecht: Springer Netherlands (2002). p. 155-71. doi: 10.1007/978-94-010-0448-0_11

18. Patti GJ, Yanes O, Siuzdak G. Metabolomics: the apogee of the omics trilogy. Nat Rev Mol Cell Biol. (2012) 13:263-9. doi: 10.1038/nrm3314

19. Armitage EG, Barbas C. Metabolomics in cancer biomarker discovery: current trends and future perspectives. J Pharm Biomed Anal. (2014) 87:111. doi: 10.1016/j.jpba.2013.08.041

20. Campisi J. Aging, cellular senescence, and cancer. Annu Rev Physiol. (2013) 75:685-705. doi: 10.1146/annurev-physiol-030212-183653

21. Domingo-Almenara X, Siuzdak G. Metabolomics Data Processing Using XCMS. In: Li S, editor. Computational Methods and Data Analysis for Metabolomics. New York, NY: Springer US (2020). p. 11-24. doi: 10.1007/978-1-0716-0239-3_2

22. Smith CA, Want EJ, O'Maille G, Abagyan R, Siuzdak G. XCMS: processing mass spectrometry data for metabolite profiling using nonlinear peak alignment, matching, and identification. Anal Chem. (2006) 78:77987. doi: $10.1021 /$ ac051437y

23. Chong J, Wishart DS, Xia J. Using MetaboAnalyst 4.0 for Comprehensive and integrative metabolomics data analysis. Curr Protoc Bioinforma. (2019) 68:1-128. doi: $10.1002 / \mathrm{cpbi} .86$

24. Xia J, Wishart DS. Web-based inference of biological patterns, functions and pathways from metabolomic data using MetaboAnalyst. Nat Protoc. (2011) 6:743-60. doi: 10.1038/nprot.2011.319

25. Schrimpe-Rutledge AC, Codreanu SG, Sherrod SD, McLean JA. Untargeted Metabolomics strategies-challenges and emerging directions. J Am Soc Mass Spectrom. (2016) 27:1897-905. doi: 10.1007/s13361-016-1469-y

26. Chong J, Soufan O, Li C, Caraus I, Li S, Bourque G, Wishart DS, et al. MetaboAnalyst 4.0: towards more transparent and integrative metabolomics analysis. Nucleic Acids Res. (2018) 46:W486-94. doi: 10.1093/nar/gky310

27. Li S, Park Y, Duraisingham S, Strobel FH, Khan N, Soltow QA, et al. Predicting network activity from high throughput metabolomics. PLoS Comput Biol. (2013) 9:e1003123. doi: 10.1371/journal.pcbi.1003123

28. Chong J, Xia J. Using MetaboAnalyst 4.0 for metabolomics data analysis, interpretation, and integration with other omics data. In: $\mathrm{Li}$ S, editor. Computational Methods and Data Analysis for Metabolomics. Methods in Molecular Biology. New York, NY: Springer US. p. 337-60. doi: 10.1007/978-1-0716-0239-3_17

29. Subramanian A, Tamayo P, Mootha VK, Mukherjee S, Ebert BL, Gillette MA, et al. Gene set enrichment analysis: a knowledge-based approach for interpreting genome-wide expression profiles. Proc Natl Acad Sci USA. (2005) 102:15545-50. doi: 10.1073/pnas.0506580102

30. Pang Z, Chong J, Li S, Xia J. MetaboAnalystR 3.0: toward an optimized workflow for global metabolomics. Metabolites. (2020) 10:186. doi: 10.3390/metabo10050186

31. Mello FW, Melo G, Kammer PV, Speight PM, Rivero ERC. Prevalence of odontogenic cysts and tumors associated with impacted third molars: a systematic review and meta-analysis. J Cranio-Maxillofacial Surg. (2019) 47:996-1002. doi: 10.1016/j.jcms.2019.03.026

32. Ventä I, Kylätie E, Hiltunen K. Pathology related to third molars in the elderly persons. Clin Oral Investig. (2015) 19:1785-9. doi: 10.1007/s00784-014-1 395-y

33. Kautto A, Vehkalahti MM, Ventä I. Age of patient at the extraction of the third molar. Int J Oral Maxillofac Surg. (2018) 47:947-51. doi: 10.1016/j.ijom.2018.03.020

34. Adelsperger J, Campbell JH, Coates DB, Summerlin D-J, Tomich CE. Early soft tissue pathosis associated with impacted third molars without pericoronal radiolucency. Oral Surg Oral Med Oral Pathol Oral Radiol Endodontol. (2000) 89:402-6. doi: 10.1016/S1079-2104(00)70119-3

35. Galluzzi L, Yamazaki T, Kroemer G. Linking cellular stress responses to systemic homeostasis. Nat Rev Mol Cell Biol. (2018) 19:731-45. doi: 10.1038/s41580-018-0068-0

36. Daley TD, Wysocki GP. The small dentigerous cyst. Oral Surg Oral Med Oral Pathol Oral Radiol Endodontol. (1995) 79:77-81. doi: 10.1016/S1079-2104(05)80078-2

37. Kim J, Ellis GL. Dental follicular tissue: misinterpretation as odontogenic tumors. J Oral Maxillofac Surg. (1993) 51:7627. doi: $10.1016 / \mathrm{S} 0278-2391(10) 80417-3$

38. de Mello Palma V, Danesi CC, Arend CF, Venturini AB, Blaya DS, Neto MM, et al. Study of pathological changes in the dental follicle of disease-free impacted third molars. J Maxillofac Oral Surg. (2018) 17:6115. doi: 10.1007/s12663-018-1131-2

39. Wood CL, Lane LC, Cheetham T. Puberty: normal physiology (brief overview). Best Pract Res Clin Endocrinol Metab. (2019) 33:101265. doi: 10.1016/j.beem.2019.03.001

40. Ackermann D, Groessl M, Pruijm M, Ponte B, Escher G, D’Uscio CH, et al. Reference intervals for the urinary steroid metabolome: the impact of sex, age, day and night time on human adult steroidogenesis. PLoS ONE. (2019) 14:e0214549. doi: 10.1371/journal.pone.0214549

41. Schiffer L, Barnard L, Baranowski ES, Gilligan LC, Taylor AE, Arlt $\mathrm{W}$, et al. Human steroid biosynthesis, metabolism and excretion are differentially reflected by serum and urine steroid metabolomes: a comprehensive review. J Steroid Biochem Mol Biol. (2019) 194:105439. doi: 10.1016/j.jsbmb.2019.105439 
42. Van Cauter E. Diurnal and ultradian rhythms in human endocrine function: a minireview. Horm Res. (1990) 34:45-53. doi: 10.1159/000181794

43. Xie G, Wang Y, Wang X, Zhao A, Chen T, Ni Y, et al. Profiling of serum bile acids in a healthy Chinese population using UPLC-MS/MS. J Proteome Res. (2015) 14:850-9. doi: 10.1021/pr500920q

44. Thomas C, Pellicciari R, Pruzanski M, Auwerx J, Schoonjans K. Targeting bile-acid signalling for metabolic diseases. Nat Rev Drug Discov. (2008) 7:67893. doi: $10.1038 / \mathrm{nrd} 2619$

45. Frommherz L, Bub A, Hummel E, Rist MJ, Roth A, Watzl B, et al. Agerelated changes of plasma bile acid concentrations in healthy adultsresults from the cross-sectional KarMeN study. PLoS ONE. (2016) 11:e0153959. doi: 10.1371/journal.pone.0153959

46. Yang J, Lu X, Liu S, Zhao S. The involvement of genes related to bile secretion pathway in rat tooth germ development. J Mol Histol. (2020) 51:99107. doi: 10.1007/s10735-020-09861-0

47. Song X, Bao M, Li D, Li YM. Advanced glycation in dgalactose induced mouse aging model. Mech Ageing Dev. (1999) 108:239-51. doi: 10.1016/S0047-6374(99)00022-6

48. Li H, Zheng L, Chen C, Liu X, Zhang W. Brain senescence caused by elevated levels of reactive metabolite methylglyoxal on D-galactose-induced aging mice. Front Neurosci. (2019) 13:1-11. doi: 10.3389/fnins.2019.01004

49. Shwe T, Bo-Htay C, Leech T, Ongnok B, Jaiwongkum T, Kerdphoo S, et al. $\mathrm{D}$-galactose-induced aging does not cause further deterioration in brain pathologies and cognitive decline in the obese condition. Exp Gerontol. (2020) 138:111001. doi: 10.1016/j.exger.2020.111001

50. Zhou YY, Ji XF, Fu JP, Zhu XJ, Li RH, Mu CK, et al. Gene transcriptional and metabolic profile changes in mimetic aging mice induced by d-galactose. PLoS ONE. (2015) 10:1-16. doi: 10.1371/journal.pone.0132088

51. He S, Sharpless NE. Senescence in health and disease. Cell. (2017) 169:100011. doi: 10.1016/j.cell.2017.05.015

52. Campisi J, d'Adda di Fagagna F. Cellular senescence: when bad things happen to good cells. Nat Rev Mol Cell Biol. (2007) 8:729-40. doi: 10.1038/nrm2233

53. Hernandez-Segura A, Nehme J, Demaria M. Hallmarks of cellular senescence. Trends Cell Biol. (2018) 28:436-53. doi: 10.1016/j.tcb.2018.02.001

54. Azman KF, Zakaria R. d-Galactose-induced accelerated aging model: an overview. Biogerontology. (2019) 20:763-82. doi: 10.1007/s10522-019-09837-y

55. Umbayev B, Askarova S, Almabayeva A, Saliev T, Masoud A-R, Bulanin D. Galactose-induced skin aging: the role of oxidative stress. Oxid Med Cell Longev. (2020) 2020:1-15. doi: 10.1155/2020/7145656

56. Cronan JE. Progress in the enzymology of the mitochondrial diseases of lipoic acid requiring enzymes. Front Genet. (2020) 11:1-6. doi: 10.3389/fgene.2020.00510

57. Mayr JA, Feichtinger RG, Tort F, Ribes A, Sperl W. Lipoic acid biosynthesis defects. J Inherit Metab Dis. (2014) 37:55363. doi: 10.1007/s10545-014-9705-8

58. Moura F, de Andrade K, Farias dos Santos J, Fonseca Goulart M. Lipoic acid: its antioxidant and anti-inflammatory role and clinical applications. Curr Top Med Chem. (2015) 15:458-83. doi: 10.2174/1568026615666150114161358

59. Molz P, Schröder N. Potential therapeutic effects of lipoic acid on memory deficits related to aging and neurodegeneration. Front Pharmacol. (2017) 8:849. doi: 10.3389/fphar.2017.00849

60. Wise GE, Frazier-Bowers S, D'Souza RN. Cellular, molecular, and genetic determinants of tooth eruption. Crit Rev Oral Biol Med. (2002) 13:32335. doi: 10.1177/154411130201300403

61. Almonaitiene R, Balciuniene I, Tutkuviene J. Factors influencing permanent teeth eruption. Part one-general factors. Stomatologija. (2010) 12:67-72.

62. Rattray NJW, Deziel NC, Wallach JD, Khan SA, Vasiliou V, Ioannidis JPA, et al. Beyond genomics: understanding exposotypes through metabolomics. Hum Genomics. (2018) 12:4. doi: 10.1186/s40246-018-0134-x

63. Robinson O, Carter AR, Ala-Korpela M, Casas JP, Chaturvedi N, Engmann J, et al. Metabolic profiles of socio-economic position: a multi-cohort analysis. Int J Epidemiol. (2020) 1-15. doi: 10.1093/ije/ dyaa 188

64. Kjær I. Mechanism of human tooth eruption: review article including a new theory for future studies on the eruption process. Scientifica. (2014) 2014:1-13. doi: 10.1155/2014/341905

65. Martincorena I, Roshan A, Gerstung M, Ellis P, Van Loo P, McLaren S, et al. High burden and pervasive positive selection of somatic mutations in normal human skin. Science (80- ). (2015) 348:880-6. doi: 10.1126/science. aaa6806

66. Lac V, Nazeran TM, Tessier-Cloutier B, Aguirre-Hernandez R, Albert A, Lum A, et al. Oncogenic mutations in histologically normal endometrium: the new normal? J Pathol. (2019) 249:173-81. doi: 10.1002/path.5314

67. Marino-Enriquez A, Fletcher CDM. Shouldn't we care about the biology of benign tumours? Nat Rev Cancer. (2014) 14:701-2. doi: 10.1038/nrc3845

68. Coura BP, Bernardes VF, de Sousa SF, França JA, Pereira NB, Pontes HAR, et al. KRAS mutations drive adenomatoid odontogenic tumor and are independent of clinicopathological features. Mod Pathol. (2019) 32:799806. doi: 10.1038/s41379-018-0194-4

69. Coura BP, Bernardes VF, de Sousa SF, Diniz MG, Moreira RG, de Andrade BAB, et al. Targeted next-generation sequencing and allele-specific quantitative PCR of laser capture microdissected samples uncover molecular differences in mixed odontogenic tumors. J Mol Diagnostics. (2020) 22:13939. doi: 10.1016/j.jmoldx.2020.08.005

70. Coura BP, de Resende TAC, de Menezes VCB, Bernardes VF, de Sousa SF, Diniz MG, et al. Assessing pathogenic mutations in dental follicles as an attempt to identify early events in odontogenic tumours tumourigenesis. Arch Oral Biol. (2020) 113:104523. doi: 10.1016/j.archoralbio.2019.104523

71. de Menezes VCB, Siqueira EC de, Costa SF dos S, de Souza FTA, de Souza RP, Gomez RS, et al. Effects of aging on DNA hydroxymethylation and methylation in human dental follicles. Arch Oral Biol. (2020) 118:104856. doi: 10.1016/j.archoralbio.2020.104856

72. Dai Z, Ramesh V, Locasale JW. The evolving metabolic landscape of chromatin biology and epigenetics. Nat Rev Genet. (2020) 21:73753. doi: 10.1038/s41576-020-0270-8

73. Yang C-H, Andrew Pospisilik J. Polyphenism - a window into geneenvironment interactions and phenotypic plasticity. Front Genet. (2019) 10:132. doi: 10.3389/fgene.2019.00132

74. Kelly AD, Breitkopf SB, Yuan M, Goldsmith J, Spentzos D, Asara JM. Metabolomic profiling from formalin-fixed, paraffin-embedded tumor tissue using targeted LC/MS/MS: application in sarcoma. PLoS ONE. (2011) 6:e25357. doi: 10.1371/journal.pone.0025357

75. Donczo B, Guttman A. Biomedical analysis of formalin-fixed, paraffinembedded tissue samples: the Holy Grail for molecular diagnostics. J Pharm Biomed Anal. (2018) 155:125-34. doi: 10.1016/j.jpba.2018.03.065

76. Neef SK, Winter S, Hofmann U, Mürdter TE, Schaeffeler E, Horn H, et al. Optimized protocol for metabolomic and lipidomic profiling in formalinfixed paraffin-embedded kidney tissue by LC-MS. Anal Chim Acta. (2020) 1134:125-35. doi: 10.1016/j.aca.2020.08.005

Conflict of Interest: The authors declare that the research was conducted in the absence of any commercial or financial relationships that could be construed as a potential conflict of interest.

Copyright (C) 2021 Bastos, Vitório, Martins-Chaves, Leite-Lima, Lebron, Moreira, Duarte-Andrade, Pereira, Santos, Lange, Macedo, Canuto, Gomes and Gomez. This is an open-access article distributed under the terms of the Creative Commons Attribution License (CC BY). The use, distribution or reproduction in other forums is permitted, provided the original author(s) and the copyright owner(s) are credited and that the original publication in this journal is cited, in accordance with accepted academic practice. No use, distribution or reproduction is permitted which does not comply with these terms. 